\section{Polio eradication, and after ...}

\section{Erradicação do polio, e depois ...}

\section{GJ Ebrahim 1}

${ }^{1}$ Department of International Child Health. Institute of Child Health. University of London. 30 Guilford Street. London WCIN 1EH. UK
Humans are the only natural reservoir of the poliovirus, and since a chronic carrier state does not occur, interruption of poliovirus transmission holds the promise of eradication. Ever since the advent of the vaccine against polio, full eradication has been the long-term goal of the international community. ${ }^{1}$ Knowledge of the techniques of tissue culture made production of vaccine possible. Vaccine virus was grown in cultures of monkey kidney cells. Treatment with formaldehyde eliminated virus infectivity whilst retaining immunogenicity. Large scale field trials showed that $80 \%$ of the recipients were protected after two doses, $91 \%$ after three, and $96 \%$ after four leaving about $4 \%$ unprotected. ${ }^{2}$ Overall protection was less for Type I virus (60\% to $70 \%$ ), compared to Types II and III (> 90\%), due to the pre-sence of thimerosol used as preservative, which was soon replaced. The Cutter incident ${ }^{3}$ due to presence of aggregates in the virus harvests that did not get inactivated by formaldehyde was a temporary setback, which was rectified by adding a filtration step to remove agglutinated virus in the manufacturing process. During the past 40 years several countries have used the inactivated polio vaccine (IPV) to control poliomyelitis. 4

Repeated passage in tissue culture yielded populations of the poliovirus that showed attenuation. $\mathrm{Pu}-$ rification resulted in clones of all three types that were attenuated to the point of minimal neurotropism in monkeys. Oral administration of these strains reproduced the natural infection capable of inducing resistance to infection comparable to the immune host. Interestingly, pharyngeal secretion of the vaccine virus was detected in two-thirds of the recipients, and there were even higher rates of faecal excretion lasting upward of 137 days. ${ }^{1}$ Soon oral polio vaccine (OPV) came to be preferred because of ease of administration, expected long-lasting immunity and production of bowel immunity leading to interruption of the chain of transmission, and spread of the vaccine virus in the community. 5

Several problems came to light with the widescale use of OPV. It has poor thermal stability under storage and field conditions requiring careful maintenance of a cold chain. In developing countries immunization with OPV produced less than optimal rates of sero-conversion, partly due to the thermal instability of the vaccine and partly because of competing enteroviruses in the environment. Thirdly, OPV has the potential hazard of reverting to a virulent form. Vaccine virus excreted by the recipients are known to mutate, and become capable of causing paralytic disease in the vaccinees or family contacts. 6 The overall risk of vaccine associated paralysis has been estimated to be one per 2,5 million doses of OPV. The risk is 9,7 times higher for the first dose than after all subsequent OPV doses with an estimated risk of one case per 520.000 doses for the first dose and one per 12,3 million doses for subsequent doses. ${ }^{7}$

Since 1962 Cuba has carried out regular mass campaigns as the only method of immunizing children against polio. 8 Similarly, since 1961 Hungary has succeeded in controlling polio with annual mass campaigns, administering three doses of monovalent oral polio vaccine spaced one month apart. 9 This method involves saturating a defined geographical area with vaccine over a short period creating sud- 
den rise of herd immunity, and blocking transmission of wild type polio virus. The strategy was further refined in Brazil, 10 and is now evolved into National Immunization Days (NIDs). Herd immunity generated through mass campaigns appears to be superior to that produced by routine immunization. ${ }^{11}$

These experiences have led to the current strategy of eradication as recommended by the World Health Organization (WHO) viz.

High routine immunization coverage. Countries should aim to vaccinate at least $90 \%$ of infants against polio by one year of age through routine immunization services. (Four doses of trivalent OPV at birth, and at 6,10 and 14 weeks of age).

National Immunization days. All children < five years of age be immunized regardless of prior immunization status. Two rounds are conducted between four to six weeks apart to be accomplished in as short a time as possible. Interrupting transmission is easiest when seasonal factors are not conducive for spread of wild poliovirus.

Acute Flaccid Paralysis (AFP) surveillance. Eradication requires that sensitive surveillance systems capable of identifying cases of polio be in place in every country. Data from the AFP surveillance system are required to certify eradication. As part of the AFP surveillance, clinical and epidemiological data and two stool samples are collected from each reported case. The stool samples must be transported under refrigeration to a laboratory certified by WHO as part of global polio laboratory network.

Mopping up. Based on surveillance data the pockets of persisting wild virus transmission are identified for intensive local immunization campaigns. Mopping-up campaigns immunize all children < five years of age in the target area regardless of their prior immunization status. Two doses of OPV are administered one month apart.

The four-pronged strategy for eradication has been remarkably successful. In 1994 the Western hemisphere (WHO region of the Americas) was certified polio free. The WHO European region (made up of 51 countries) has not had any new cases of indigenous polio for almost two years. More recently, October 2000, the WHO Western Pacific region (which includes 37 countries ranging from China to several island nations) has been certified polio free. Approximately 50 per cent of the world's population reside in areas that are polio free. However, two large endemic areas of continued poliovirus transmission exist in South Asia and sub-Saharan Africa. Seven reservoir countries (Bangladesh, Democratic Republic of Congo, Ethiopia, India, Nepal, Nigeria and Pakistan) and seven countries in conflict
(Afghanistan, Angola, Liberia, Sierra Leone, Somalia, Sudan, and Tajikistan) are to be targeted for accelerated implementation of the four-pronged strategy of eradication. 12

Application of genomic sequences to virological surveillance has revealed the existence of multiple genotypes of wild polioviruses in various regions of the world. These have been greatly reduced in number as global eradication has progressed. Each genotype is believed to represent an independent reservoir of poliovirus transmission, and each can be used as a pointer to a geographically defined target area in which immunization coverage must be improved.

As the target of global eradication increasingly appears achievable, questions of current immunization policies in the polio free regions are being addressed. There is the ever present risk of re-introduction of wild poliovirus into the polio free regions from countries where polio is endemic. 13 On the other hand, vaccine associated paralysis, however rare, poses a comparatively greater danger than the disease. Sabin strains differ from their wild type parents by just 10 nucleotides, and mutated vaccine derived polio virus that are neurovirulent in animal models can be isolated from the stools of children within days of vaccination. ${ }^{14}$ Hence safer forms of vaccination as well as surveillance must continue into the foreseeable future in all regions certified as polio free.

The development of enhanced potency inactivated polio vaccine (eIPV) ${ }^{15}$ opens up the way to a new strategy of immunization in the interim period as the countdown to full eradication proceeds. Trials in several countries (three in United Sates and 15 elsewhere) have established the immunogenicity of two doses of eIPV in the first year of life. 16 In those who receive OPV, previous immunization with IPV can prevent excretion of poliovirus from the pharynx and reduces the duration and degree of excretion in the stool.17 Moreover, incorporation of at least one dose of eIPV at the start of the immunization schedule tends to increase not only the systemic but mucosal antibody production. The safety of sequential schedules of one to three doses of IPV followed by one to three doses of OPV has been assessed in several studies with no adverse effects reported.18 Hence sequential eIPV/OPV is the recommended schedule for immunization in the interim period to full global eradication.

In the post eradication era the continuing circulation of vaccine derived polioviruses (VDPV) cannot be completely ruled out. In Cuba and Hungary, both of which have used mass campaigns with OPV as the only method of immunizing children against 
polio, enterovirus surveillance has recovered polioviruses for only one months and three months respectively following the campaigns. Whether VDPV circulation would be more persistent in the unhygienic environments of overcrowded urban slums and shanty towns needs to be investigated. Even if no persistence of VDPV is detected the likelihood of emergence and spread from reservoirs like immunocompromised individuals when herd immunity in the population has declined cannot be discounted. If VDPV do not persist then stopping immunization is the obvious next step, with a possible saving of $\$ 1.5$ billion on vaccine alone. If vaccine strains do persist then continuing immunization in those at highest risk of persistent circulation with inactivated polio vaccine may be needed for the foreseeable future, or until newer vaccines are developed.

Policy decisions also need to be made about containment of the virus held in stock. Experience with regard to stocks of small pox and vaccinia viruses following the eradication of small pox can be a useful guide.

\section{References}

1. Sabin AB. Oral poliovirus vaccine. History of its development and use and current challenge to eliminate poliomyelitis from the world. J Infect Dis 1985; 151: 420-36.

2. Salk J, Drucker JA, Malvy D. Non-infectious poliovirus vaccines. In: Plotkin SA, Mortimer DA, editors. Vaccines. 2nd ed. Philadelphia: WB Saunders; 1994. p. 295.

3. Nathanson N, Longmuir AD. The Cutter incident. Poliomyelitis following following formaldehyde - inactivated poliovirus vaccination in the United States during the spring of 1955. II and III. Relationship of poliomyelitis to Cutter vaccine. Am J Hyg 1963; 78: 29-60.

4. Murdin AD, Barreto L, Plotkin S. Inactivated poliovirus vaccine: past and present experience. Vaccine 1996; 14: 735-46.

5. Nathanson N. Eradication of poliomyelitis in the United States. Rev Infect Dis 1982; 4: 940-50.

6. Hinman AR, Koplan JP, Oranstein WA, Brink EW, Nkowane BM. Live or inactivated poliomyelitis vaccine: an analysis of benefits and risks. Am J Pub Health 1988; 78: 291-5.

7. Nkowane BM, Wassilack SGF, Orenstein WA, Bart KJ, Schnberger LB, Hinman AR, Kew OM. Vaccine associated paralytic poliomyelitis, United States, 1973 through 1984. JAMA 1987; 257: 1335-40.

8. Ochoa EG, Lago PM. Epidemiological surveillance and control of poliomyelitis in the Republic of Cuba. J Hyg Epidemiol Microbiol Immunol 1987; 31: 381-9.

9. Domok I. Experiences associated with the use of the live poliovirus vaccine in Hungary 1959-1962. Rev Infect Dis 1984; 6 Suppl: S413-8
10. Risi JP. The control of poliomyelitis in Brazil. Rev Infect Dis 1984; 6 Suppl: S400-3.

11. Richardson G, Linkins RW, Eames MA, Wood DJ, Campbell PJ, Ankers E, Deniel M, Kabbaj A, Magrath DI, Minor PD. Immunogenicity of oral poliovirus vaccine administered in mass campaigns versus routine immunization programmes. Bull World Health Organ 1995; 73: 769-77.

12. Centers for Disease Control and Prevention. Progress towards global poliomyelitis eradication, 1997-1998. Mor Mortal Wkly Rep 1999; 48: 416-21.

13. Centers for Disease Control and Prevention. Importation of wild poliovirus into Qinghai Province, China 1999. JAMA 2000; 283: 1414-5.

14. Montagnon BJ, Fanget B, Vincent-Falquot JC. Industria scale production of inactivated poliovirus vaccine prepared by culture of Vero cells on microcarriers. Rev Infect Dis 1984; 6 (S2): S341-4.

15. Vidor E, Meschievitz C, Plotkin S. Fifteen years of experience with Vero produced enhanced potency inactivated poliovirus vaccine. Pediatr Infect Dis J 1997; 16: 312 22

16. Faden H. Poliovirus vaccination: a trilogy. J Infect Dis 1993; 168: $25-8$ 